\title{
Информатика, вычислительная
}

техника и управление

УДК 625.739.4:625.712.1:656.11

DOI: $10.12737 /$ article_59b11cbf31cbf2.58347936

С.П. Шец, Е.В. Справцева, А.А. Калмыков

\section{ПРИМЕНЕНИЕ ИМИТАЦИОННОГО МОДЕЛИРОВАНИЯ ПРИ СОВЕРШЕНСТВОВАНИИ ОРГАНИЗАЦИИ ДОРОЖНОГО ДВИЖЕНИЯ НА ПЕРЕКРЕСТКЕ ГОРОДА БРЯНСКА}

Проведен анализ существующей схемы организации дорожного движения на пересечении улиц Объездной, Авиационной и переулка Пилотов в городе Брянске. Выявлены недостатки, а также предложены мероприятия по проектированию кольцевой развязки на перекрестке. Разработаны имитационные модели базового и проектного вари- антов перекрестка в среде PTV Vissim. Проведена оценка эффективности введения кругового движения.

Ключевые слова: кольцевое пересечение, имитационное моделирование, транспортный поток, организация дорожного движения, регулируемый перекресток, задержки транспорта.

\section{S.P. Shets, E.V. Spravtseva, A.A. Kalmykov}

\section{SIMULATION APPLICATION AT IMPROVEMENT OF TRAFFIC ORGANIZATION ON CROSSROADS OF BRYANSK}

The paper reports the considered option of designing the circuit of a ring motion on the controlled crossroads in the city of Bryansk. For comparison of the basic option with the designed one the application of the simulation in the PTV Vissim environment is carried out.

To the advantages of a circular motion in contrast to a motion controlled with traffic lights one can refer the decrease of accident risks and particularly the severity of traffic accident consequences as a result of a traffic speed decrease on a circular traffic way and also an accompanying character of a vehicular traffic which excludes completely accident risks with the most terrible consequences - head-on collisions. A selfcontrolled and more even thoroughfare of circular crossroads contributes to the decrease of petrol consumption and toxicity of impurities rejection coming into atmosphere with motor cars exhaust gases.

\section{Введение}

В условиях роста уровня автомобилизации существенно повышается значение сбалансированного использования ресурсов для эффективной организации дорожного движения. При этом на участках высокой интенсивности движения основной ресурс - земельный - является всегда дефицитным и одним из наиболее финансово емких. Современным инструментом сбалансированного использования ресурсов и является транспортное планирование
The simulation with the aid of the PTV Vissim software complex is a high efficient innovation solution in the field of the organization and control of street traffic and gives a possibility of the preliminary comparison of different options both, present and designed without considerable costs.

As a result of the simulation of two circuits of traffic organization there was established that the introduction of a circular traffic on the crossroads of Obiezdnaya and Aviatsionnaya Str., and Pilotov Lane in the city of Bryansk will allow decreasing considerably transport delays in comparison with traffic lights control.

Key words: ring crossroads, simulation, traffic, traffic organization, crossroads controlled, transport delays.

[1]. Анализ зарубежной практики проектирования, строительства и эксплуатации автомобильных дорог показывает, что одним из направлений повышения транспортно-эксплуатационных качеств пересечений автомобильных дорог в одном уровне является широкое применение планировочных решений кольцевой организации движения. Подобные решения применяются как на загородных автомобильных дорогах, так и на улично-дорожной сети на- 
селенных пунктов [2]. Распространение кольцевых пересечений за рубежом обусловлено их более высокими транспортноэксплуатационными качествами по сравнению с другими планировочными решениями по организации движения на пересечениях в одном уровне [3]. Применение специализированных программных приложений в настоящее время становится неотъемлемой составляющей проектирования транспортной инфраструктуры [4].
Мероприятия по использованию имитационного компьютерного моделирования транспортных и пешеходных потоков могут быть реализованы в кратчайшее время с минимальными затратами по сравнению с дорожным строительством, что позволит максимально использовать возможности имеющейся УДС и максимально учитывать потребности участников дорожного движения [5].

\section{Анализ существующей организации дорожного движения на перекрестке}

В настоящее время основные магистрали транспортной сети города Брянска перегружены транспортным потоком, в часы пик наблюдаются постоянные заторы, сопровождающиеся снижением средней скорости движения транспорта. Это приводит к увеличению суммарных затрат времени на ожидание выполнения транспортных операций, транспортной усталости населения, увеличению числа дорожно-транспортных происшествий, ухудшению экологии не только по токсичности атмосферы, но и по шуму от движущихся потоков автомобилей. Основными причинами заторов в Брянске, как и в других городах, являются нерациональная общая транспортная схема города, малая пропускная способность УДС, нерациональная организация движения, нарушение правил парковки водителями транспортных средств, ДТП на дороге и т.д.

Рассматриваемый перекресток улиц Объездной, Авиационной и переулка Пилотов в городе Брянске является регулируемым, продолжительность цикла -87 с, в выделенную пешеходную фазу осуществляется движение пешеходов по двум пешеходным переходам. Пересечение характеризуется высокоинтенсивным движением транспортных потоков. Исследованиями установлено, что в утренний и вечерний пиковые периоды интенсивность превышает 2500 авт./ч, при этом суммарная численность пешеходов, переходящих проезжие части ул. Авиационной и пер. Пилотов, не превышает 500 чел./ч (рис. 1).
На картограмме транспортных и пешеходных потоков можно выделить интенсивный левоповоротный поток со стороны улицы Объездной. В составе потока присутствуют автомобили разных типов: $82,6 \%$ - легковые автомобили, почти 3,5\% - грузовые транспортные средства, чуть более $13 \%$ - автобусы различной вместимости и примерно $1 \%$ троллейбусов.

Анализ дорожной ситуации на перекрестке позволил выявить значительные очереди на всех подходах к перекрестку в пиковые периоды.

Для повышения пропускной способности и снижения транспортных задержек на перекрестке предлагается разработать проект кругового движения на перекрестке с диаметром центрального островка 30 м и двумя полосами на кольцевой проезжей части.

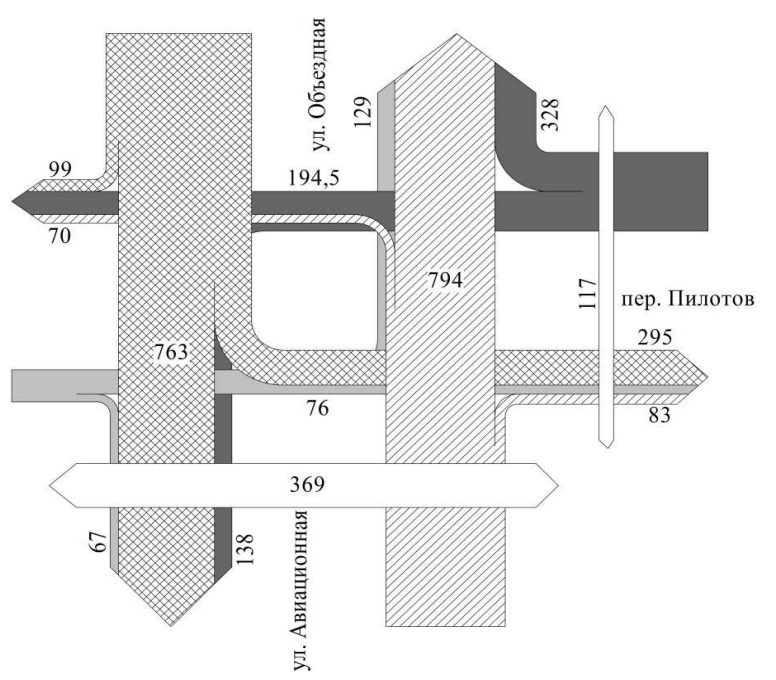

Рис. 1. Картограмма интенсивности транспортных и пешеходных потоков в пиковый вечерний период 


\section{Преимущества кругового движения}

Общепризнано снижение аварийности и особенно тяжести дорожнотранспортных происшествий на кольцевых пересечениях, что обусловлено пониженными скоростями движения в зоне кольцевых пересечений и меньшей разницей между максимальными и минимальными скоростями транспортных средств при проезде пересечений [2]. Правильно запроектированная развязка с круговым движением полностью исключает риск ДТП с наиболее тяжкими последствиями - лобовых столкновений (в случае возникновения аварии последствия, как правило, ограничиваются лишь повреждениями кузова автомобиля). Также при въезде на пересечение водителям необходимо контролировать только одно направление, в результате чего при выполнении маневра водители не испытывают психологического напряжения из-за необходимости следить за автомобилями, движущимися с других направлений, ожидая разрыва в потоке, дос-

\section{Применение имитационного моделирования}

В сложившейся ситуации, при отсутствии возможности частичной или полной реконструкции УДС города (плотная городская застройка, необходимость обеспечения безопасности движения пешеходов и т.д.), необходима разработка высокоэффективных инновационных решений в сфере организации и управления дорожным движением. Одним из решений является моделирование существующих и прогнозируемых транспортных и пешеходных потоков при помощи программного комплекса PTV Vissim для осуществления оперативного и стратегического планирования работы светофорных объектов и оценки эффективности схем организации движения.

Возможности программы PTV Vissim позволяют осуществлять:

- моделирование перекрестков, регулируемых правилами приоритета или светофорами, транспортных развязок разных уровней сложности;

- визуализацию дорожного и пешеходного движения; таточного для въезда на пересечение. Это обстоятельство обеспечивает более высокую пропускную способность кольцевых пересечений по сравнению с нерегулируемыми.

Саморегулируемый и более равномерный проезд кольцевых пересечений способствует снижению расхода горючего и токсичности выбросов вредных веществ, поступающих в атмосферу с отработавшими газами автомобилей. Расход горючего при этом сокращается более чем на $25 \%$, а токсичность выбросов вредных веществ (углекислый газ, оксиды азота, окись углерода) - на $15-30 \%$. В результате уменьшения количества остановок автомобилей перед проездом кольцевых пересечений, а следовательно, и необходимости разгона остановившихся автомобилей снижается транспортный шум. Считается, что можно добиться снижения транспортного шума на 2-4 дБА [3].

- анализ пропускной способности транспортных сетей;

- прогнозирование загрузки участков улично-дорожной сети и возникновения заторовых ситуаций при различных вариантах организации дорожного движения;

- оценку транспортной эффективности вариантов проектных решений;

- оптимизацию светофорных циклов при регулировании движения (определение программы светофорного регулирования исходя из минимизации средней задержки транспортных средств на всех направлениях).

Кроме того, программа позволяет определять время проезда маршрута, длину очереди, уровень шума и выхлопов вредных веществ, а также выявлять заторовые ситуации [6].

Задачи, которые последовательно решались с помощью программы PTV Vissim при разработке модели базового варианта перекрестка: 
1. Моделирование дорожного полотна с учетом геометрических параметров пересечения (рис. 2 а) с заданием соответствующих направлений движения.

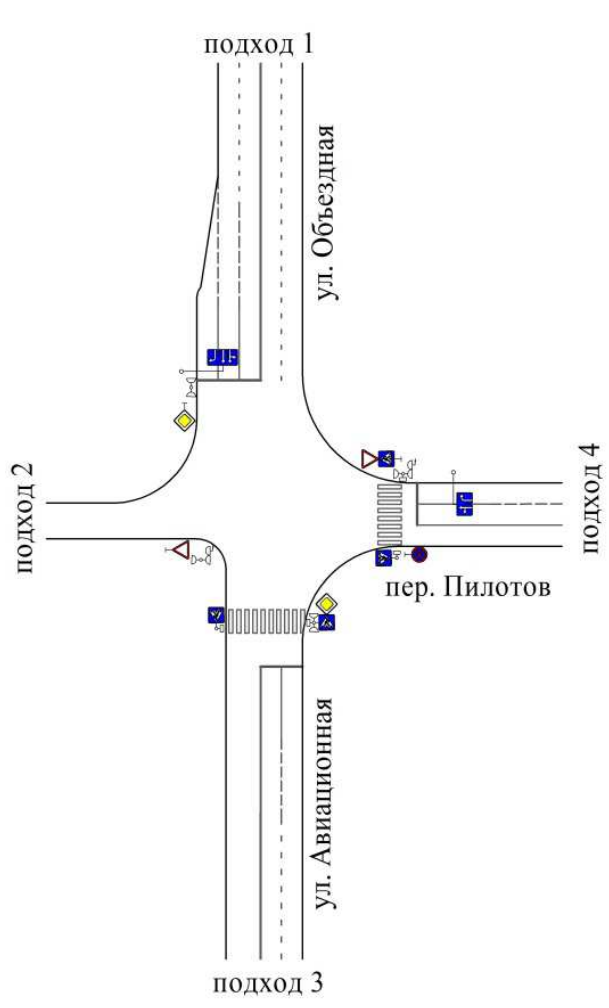

a)

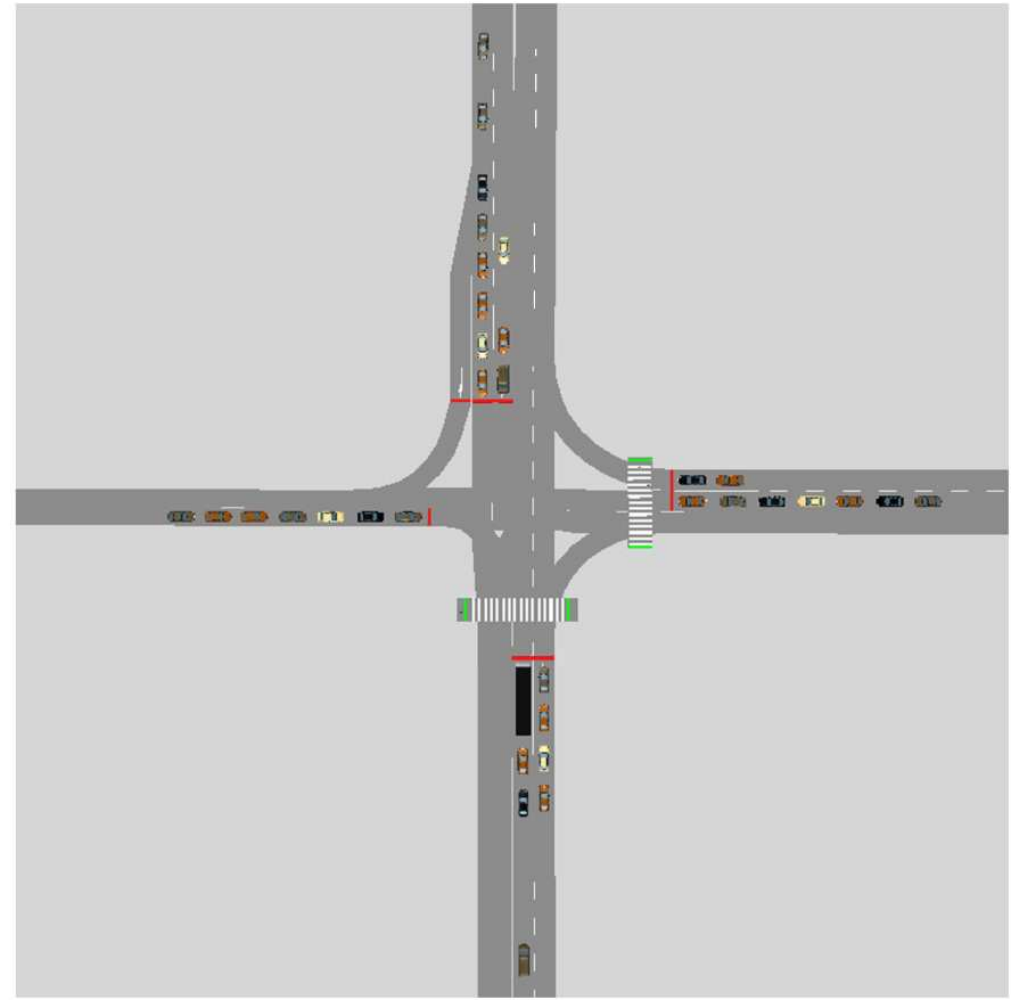

б)

Рис. 2. Базовый вариант перекрестка: а - общий вид; б - результат моделирования

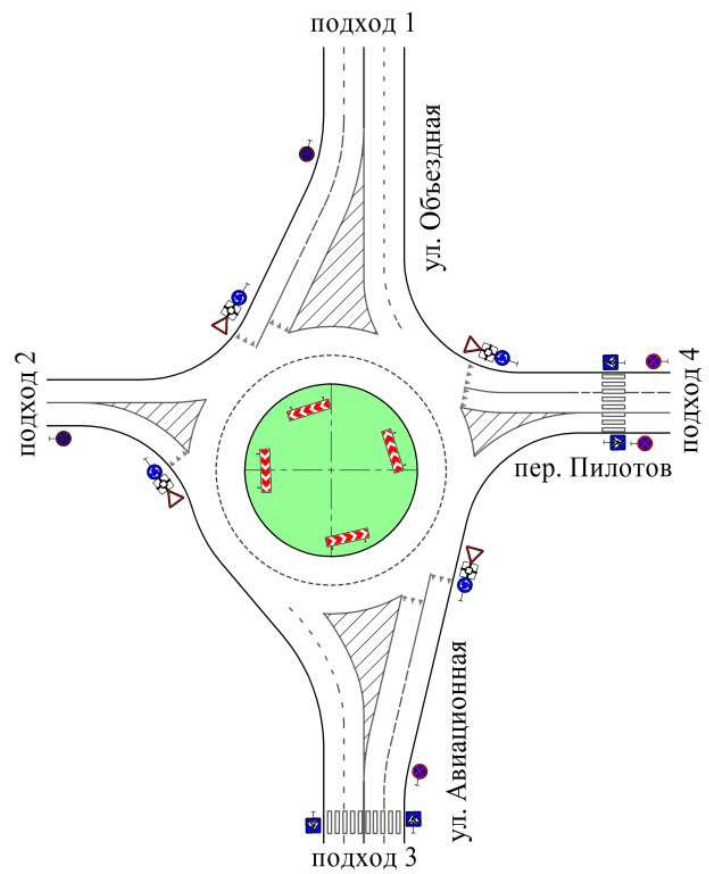

a)

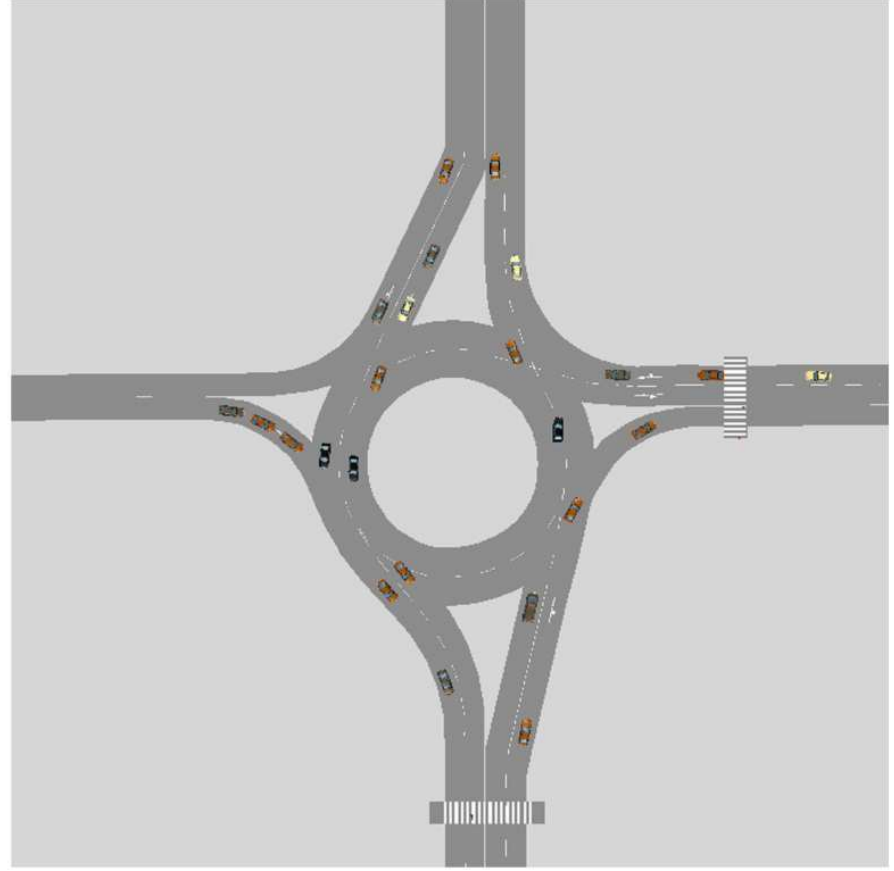

б)

Рис. 3. Проектный вариант перекрестка: а - общий вид; б - результат моделирования

2. Задание интенсивностей и состава транспортных потоков на входящих участках с распределением трафика по полосам и направлениям движения, а также задание скоростей движения каждого типа автомобилей. 
3. Нанесение на дорожное полотно пешеходных переходов, задание интенсивности и направлений движения пешеходов.

4. Введение параметров светофорного регулирования в соответствии с графиком включения сигналов светофоров и схемой пофазного разъезда.

5. Рассмотрение конфликтных зон с расстановкой приоритетов движения согласно Правилам дорожного движения.

6. Пробная имитация модели для проверки ее адекватности.

7. Устранение возникших ошибок путем доработки модели.

8. Формирование списка результатов из необходимых для анализа параметров.

При моделировании проектного варианта развязки с круговым движением последовательность разработки модели была той же, за исключением введения параметров светофорного регулирования. Отсутствие жилой застройки в непосредственной близости от перекрестка дало возможность принимать геометрические параметры въездов и выездов с кольцевого пересечения в соответствии с рекомендациями для свободных условий. При рекон- струкции участка УДС вблизи пересечения не будут затронуты жилые объекты, а также торговые площади.

С целью повышения пропускной способности на кольцевой развязке были предложены такие меры, как запрет парковки вдоль кольцевой проезжей части, а также на подходах к кольцу и перенос пешеходных переходов. Результаты проектирования и моделирования перекрестка с круговым движением представлены на рис. 3.

Сравнение результатов моделирования двух вариантов позволяет увидеть, что на кольце движение стало более плавным и равномерным, значительно снизились очереди на подходах к перекрестку. Это подтверждают результаты расчета средней задержки транспорта в программе PTV Vissim (таблица).

Так как моделирование в обоих вариантах проводилось для пикового значения интенсивности, то можно ожидать, что в другие часы суток движение на кольцевой развязке будет еще более свободным и комфортным для водителей.

Таблица

Сравнительный анализ схем организации дорожного движения

\begin{tabular}{|c|c|c|c|c|}
\hline \multirow{2}{*}{$\begin{array}{c}\text { Номер подхода } \\
\text { к перекрестку }\end{array}$} & \multicolumn{2}{|c|}{ Средняя задержка, с } & \multicolumn{2}{|c|}{$\begin{array}{c}\text { Проехало автомобилей (за } \\
\text { интервал моделирования 10 мин) }\end{array}$} \\
\cline { 2 - 5 } & $\begin{array}{c}\text { Базовый } \\
\text { вариант }\end{array}$ & $\begin{array}{c}\text { Проектный } \\
\text { вариант }\end{array}$ & $\begin{array}{c}\text { Базовый } \\
\text { вариант }\end{array}$ & $\begin{array}{c}\text { Проектный } \\
\text { вариант }\end{array}$ \\
\hline 1 & 16,88 & 7,28 & 268 & 262 \\
\hline 2 & 54,03 & 6,93 & 48 & 58 \\
\hline 3 & 25,13 & 8,94 & 222 & 220 \\
\hline 4 & 44,10 & 8,01 & 127 & 162 \\
\hline
\end{tabular}

\section{Заключение}

Строительство кольцевой развязки дорогостоящее мероприятие, поэтому целесообразно до проведения дорожностроительных работ оценить эффективность проектной схемы ОДД с помощью имитационного моделирования в среде PTV Vissim. Проведенный сравнительный

\section{СПИСОК ЛИТЕРАТУРЫ}

1. Горев, А.Э. Основы транспортного моделирования: практ. пособие / А.Э. Горев, К. Бёттгер, А.В. Прохоров, Р.Р. Гизатуллин. - СПб.: КОС- анализ базового варианта перекрестка со светофорным регулированием и проектного варианта с круговым движением показал, что при оборудовании кольцевой развязки средние задержки транспорта на подходах к перекрестку снизятся в 2,3-7,8 раза.

ТА, 2015. - 168 с. - (Серия «Библиотека транспортного инженера»). 
2. Современные кольцевые пересечения / А.В. Зедгенизов, Р.Ю. Лагерев, А.Г. Левашев, А.С. Липницкий, А.Ю. Михайлов, М.И. Шаров. Деп. 24.12.09, № 823-В2009.

3. Методическое обеспечение автоматизированного проектирования кольцевых пересечений / П.И. Поспелов, Б.А. Щит, М.А. Овчинников, А.А. Вершков, М.Н. Зобнин, А.В. Жуков, П.А. Елугачев, М.А. Катасонов, Г.В. Величко, Л.И. Сикорская // САПР и ГИС автомобильных дорог. - 2014. - № 2 (3). - С. 46-56.

4. Декина, А.И. Обзор программных средств планирования и моделирования транспортных по-

1. Gorev, A.E. Fundamentals of traffic modeling: Practical Textbook / A.E. Gorev, K. Boettger, A.V. Prokhorov, R.R. Gizatullin. - S-Pb.: KOSTA, 2015. - pp. 168. - (Series "Library of Transport Engineer").

2. Modern Ring Crossroads / A.V. Zedgenizov, R.Yu. Lagerev, A.G. Levashev, A.S. Lipnitsky, A.Yu. Mikhailov, M.I. Sharov. - Dep. 24.12.09, № 823V2009.

3. Methodical support of ring crossroads CAD / P.I. Pospelov, B.A. Shchit, M.A. Ovchinnikov, A.A. Vershkov, M.N. Zobnin, A.V. Zhukov, P.A. Yelugachev, M.A. Katasonov, G.V. Velichko, L.I. Si-

\section{Сведения об авторах:}

Шец Сергей Петрович, д.т.н., зав. кафедрой «Автомобильный транспорт» Брянского государственного технического университета, e-mail: shetssp@mail.ru.

Справцева Екатерина Викторовна, ст. преподаватель кафедры «Автомобильный транспорт»

Shets Sergey Petrovich, D. Eng., Head of the Dep. "Motor Transport", Bryansk State Technical University, e-mail: shetssp@mail.ru.

Spravtseva Catherine Victorovna, Senior lecturer of the Dep. "Motor Transport", Bryansk State Technical University, e-mail: kama3@ list.ru. токов / А.И. Декина // Информационнотелекоммуникационные системы и технологии: материалы науч.-практ. конф. - Кемерово: КузГТУ им. Т.Ф. Горбачева, 2015. - С. 148.

5. Ведяшкин, В.И. Моделирование транспортных и пешеходных потоков при помощи программного комплекса «PTV VISSIM» в г. Барнауле / В.И. Ведяшкин // Педагогическое образование на Алтае. - 2014. - № 2. - С. 219-220.

6. А+S. Краткое руководство по выполнению проектов в PTV VISSIM 6. - 2014. - 76 с.

korskaya// CAD and GIS of Highways. - 2014. - № 2 (3). - pp. 46-56.

4. Dekina, A.I. Review of software means of traffic / A.I. Dekina // Information-Telecommunication Systems and Technologies:Proceedings of the Scientific-Practical Conf. - Kemerovo: GorbachevSTU of Kuzbass. - Kemerovo: GorbachevSTU of Kuzbass, 2015. - pp. 148.

5. Vedyashkin, V.I. Simulation of traffic and pedestrian stream using "PTV VISSIM" software complex in Barnaul / V.I. Vedyashkin // Pedagogical Education in Altay. - 2014. - № 2. - pp. 219-220.

6. A+S. Short Guide on Projects Fulfillment in PTV VISSIM 6. - 2014. - pp. 76.

Статья поступила в редколлегию 19.04.17. Рецензент: д.т.н., профессор Брянского государственного технического университета Бимутин С.Г.

Брянского государственного технического университета, e-mail: kama3@ list.ru.

Калмыков Александр Анатольевич, студент Брянского государственного технического университета, e-mail: gazaza243@gmail.com.

Kalmykov Alexander Anatolievich, Student, Bryansk State Technical University, e-mail: gazaza243@gmail.com. 